\title{
VIOLÊNCIA SEXUAL CONTRA CRIANÇAS E ADOLESCENTES: RESILIÊNCIA E PROTAGONISMO NA AMAZÔNIA
}

\author{
Joaquim Hudson de Souza Ribeiro \\ Faculdade Salesiana Dom Bosco (FSDB \\ Universidade do Estado do Amazonas (UEA) \\ Programa de Pós Doutoramento da Faculdade de \\ Psicologia da Universidade do Porto (FPCEUP) \\ jhudsonmanaus@hotmail.com \\ Susana Maria Gonçalves Coimbra \\ Faculdade de Psicologia e de Ciências da Educação da \\ Universidade do Porto (FPCEUP) \\ susana@fpce.up.pt \\ Vanessa Barbosa Romeira Leme \\ Universidade do Estado do Rio de Janeiro (UERJ) \\ vanessaromera@gmail.com
}

Fecha de Recepción: 15 Junio 2019

Fecha de Admisión: 25 Septiembre 2019

\begin{abstract}
RESUMO
A literatura internacional no que concerne à temática do abuso sexual de crianças e adolescentes tem focalizado maior atenção para os impactos negativos no desenvolvimento frente às experiências abusivas sofridas, desempenhando muitas vezes o papel de vítimas passivas. Menos investigações têm procurado identificar os fatores de proteção que podem promover a resiliência entre os/as mais jovens e, em particular, o contributo que os/as próprios/as podem dar na sua identificação e acionamento. 0 presente estudo tem por objetivo apresentar a experiência do Projeto Içá-Ação e Proteção (Cáritas), com o apoio do grupo Aleimar Onlus, na prevenção da violência sexual contra crianças e adolescentes na Amazônia brasileira, identificando fatores de resiliência em ações de protagonismo de adolescentes. Trata-se de um relato de experiência das atividades desenvolvidas por 1.434 adolescentes participantes de um projeto de intervenção comunitária em 19 cidades dos estados do Amazonas e Pará no período de 2016 a 2018. Em consonância com o Eixo Protagonismo Juvenil do Plano Nacional de Enfrentamento da Violência Sexual de Crianças e Adolescentes - adotado pelo Brasil, os/as adolescentes desenvolveram ações em escolas, espaços religiosos, comunidades (indígenas, tradicionais e urbanas), Conselhos e Fóruns de participação e decisão política. As conclusões dessa experiência mostraram que resiliência não é sinônimo de invulnerabilidade e a sua promoção é imperativa para um desenvolvimento mais saudável na infância e adolescência. Os
\end{abstract}




\section{VIOLÊNCIA SEXUAL CONTRA CRIANÇAS E ADOLESCENTES: RESILIÊNCIA E PROTAGONISMO NA AMAZÔNIA}

resultados indicaram que crianças e adolescentes mais do que sujeitos passivos de qualquer ação ou programa, têm que ser assumidos/as como sujeitos de direito, de participação, decisão, de autonomia e de protagonismo participativo e transformador no enfrentamento do abuso e exploração sexual.

Palavras-chave: abuso sexual; resiliência; protagonismo; amazônia.

\section{ABSTRACT}

Sexual violence against children and adolescents: resilience and protagonism in Amazon.

International literature on the topic sexual abuse has focused more attention on the negative developmental impacts of abusive experiences, and children and adolescents involved are often perceived as passive victims. Fewer investigations have sought to identify the protective factors that can promote resilience among younger people and, in particular, the contribution they can make to their identification and use. This study aims at presenting the experience of the Projeto Içá-Ação e Proteção (Içá-Action and Protection Project) (Caritas) and Aleimar Onlus, in the prevention of sexual violence against children and adolescents in the Brazilian Amazon, identifying resilience factors in actions protagonized by the adolescents. This study is preliminary report of the activities of this experimental project carried out by 1,434 adolescents who participated in a community intervention project in 19 cities of the states of Amazonas and Pará from 2016 to 2018. In line with the Youth Protagonism Axis of the National Plan for the Fight against Sexual Violence of Children and Adolescents - adopted by Brazil, the adolescents developed actions in schools, religious spaces, communities (indigenous, traditional and urban), City councils and Forums of participation and political decision. The preliminary analysis of this project showed that resilience is not synonymous of invulnerability and its promotion is imperative for healthier development during childhood and adolescence, in particular when sexual abuse occurs. The results also indicated that children and adolescents, more than passive subjects of any action or program, must be assumed as agents of their own rights', decision, autonomy and participatory and transformative advocacy in facing sexual abuse and exploitation.

Keywords: sexual abuse; resilience; protagonism; brazilian Amazon.

\section{INTRODUÇÃO}

A literatura internacional no que concerne à temática do abuso sexual de crianças e adolescentes tem focalizado maior atenção para os impactos negativos no desenvolvimento frente às experiências abusivas sofridas, desempenhando muitas vezes o papel de vítimas passivas (e.g., Gillespie, \& Panagioti. 2019; Halpern et al., 2018; Moynihan, Pitcher, \& Saewyc, 2018). Menos investigações.) têm procurado identificar os fatores de proteção que podem promover a resiliência entre os/as mais jovens e, em particular, o contributo que os/as próprios/as podem dar na sua identificação e acionamento (Antunes \& Machado, 2013; Crawford, Wright, \& Masten, 2006; Deblinger, Runyon, \& Steer, 2014; Ungar, 2015).

A violência sexual contra crianças e adolescentes é considerada um problema de saúde pública devido ao alto índice de prevalência nessa população e aos inúmeros prejuízos causados ao seu desenvolvimento cognitivo, afetivo, social e comportamental. As consequências podem aparecer em curto ou longo prazo, ou podem ficar latentes. Pesquisas apontam que crianças e adolescentes que sofreram abuso sexual podem apresentar prejuízos graves no desenvolvimento emocional e cognitivo, comportamento hipersexualizado; quatro vezes maior possibilidade de desenvolverem transtorno de estresse pós traumático, depressão, pensamento suicida, automutilação, tentativas de suicídio e suicídio (Gillespie, \& Panagioti. 2019; Halpern et al., 2018; Habigzang \& Caminha, 2004; Habigzang, Damásio \& Koller, 2013; Moynihan et al., 2018; Silva \& Gonçalves, 2015). 
Diversos autores destacam que, no plano nacional e internacional, os estudos realizados na atualidade mostram a ampliação de pesquisas quantitativas e qualitativas voltadas para 0 dimensionamento do fenômeno da violência sexual contra crianças e adolescentes (por exemplo, abuso sexual, exploração sexual e tráfico de crianças e adolescentes para fins de exploração sexual) associado a outras formas de violência, tais como os estudos de Ribeiro, 2013; Williams \& Araújo, 2009; Bazon, 2009; Algeri \& Souza, 2006. Desse modo, observa-se maior visibilidade das vítimas de violência, assim como o surgimento de novos campos de investigação e mudanças no quadro atual configurado, podendo subsidiar ainda programas de prevenção e iniciativas que atuam nesse foco (Ribeiro, 2013; Williams \& Araújo, 2009; Bazon, 2009; Algeri \& Souza, 2006).

Na Amazônia Brasileira, os estudos com enfoque na violência sexual contra crianças e adolescentes, ainda são bastante limitados. Estudar a violência sexual contra crianças e adolescentes na Amazônia nos desafia, por um lado, ao nos depararmos com as peculiaridades da região, sobretudo, as distâncias geográficas, os meios de comunicação entre os municípios, a presença de povos indígenas, comunidades tradicionais (ribeirinhos e quilombolas), a realidade urbana das metrópoles como Manaus e Belém, que chegam a concentrar grande parte de população, sobretudo nas áreas degradantes de suas periferias (Fernandez, 2018). Por outro lado, essa realidade nos convoca a um olhar diferenciado, sobretudo do ponto de vista cultural e histórico, uma vez que parte de sua construção sócio-histórico-cultural é também oriunda da violência sexual na ocupação sexista de seu território desde a presença da coroa portuguesa (Ribeiro, 2013). Assim, quando consideramos a violência enquanto expressão das relações dispostas em uma sociedade, entendemos que a violência sexual se manifesta na conjuntura das estruturas adultocêntricas e patriarcais que ainda compõem a base de muitas interações diárias em diferentes povos e culturas (Ribeiro, 2013); Fernandez, 2018). A violência sexual é uma das manifestações dessas relações de poder. Um atentado criminoso contra os direitos humanos de crianças e adolescentes (e.g., Luo, 1998; Miller, 2018).

Em todas as fases do desenvolvimento humano existem adversidades vividas, mas também com elas, possibilidades de mudança, de cura, de transformação, "superação", aprendizado, ressignificação, (re)elaboração simbólica, progresso e ação protagonista, desde que estejam presentes alguns mecanismos de proteção interna e externa (Cowan, Cowan \& Shultz, 1996; Crawford et al., 2006; Masten, 2014; Theron, \& Phasha, 2015; Wright, Crawford, \& Sebastian, 2007). Nesse sentindo, refletir sobre resiliência implica um olhar distante daquele linear estanque, focado em indivíduos resilientes ou não resilientes, uma vez que o conceito de resiliência exige ser compreendido como processo em diferentes situações, condições, fases, momentos ou contextos (Deblinger et al., 2014; Ungar, 2015; Wright, \& Masten, 2015). Assim, numa concepção bioecológica entende-se que a resiliência não depende apenas da pessoa, mas das condições internas e externas que lhes são apresentadas ou disponíveis (Narvaz \& Koller, 2004).

0 processo de resiliência ressalta, sobretudo, a pessoa no seu contexto junto ao emaranhado de relações mútuas que ela estabelece com outras pessoas, objetos e símbolos. Estas relações podem ser explicadas pelo Teoria Bioecológica do Desenvolvimento Humano (TBDH, Bronfenbrenner \& Morris, 1998) no qual o processo, a pessoa, o contexto e o tempo devem ser estudados na sua inter-relação.). Na teoria bioecológica do desenvolvimento humano, Bronfenbrenner e Morris (1998) destacam o papel dos contextos nos quais a pessoa (um ser biológico, psicológico e social) está inserida de maneira ativa e transformadora, podendo modificar suas relações sociais. Assim, a partir dos pressupostos da TBDH, estudos que focam a adolescência devem considerar tanto as características biopsicológicas, tais como mudanças físicas e 0 desenvolvimento de habilidades cognitivas e sociais, como aspectos do ambiente imediato, tais 
como relações com os pais, pares e professores e remoto, por exemplo, estereotipo de gênero, valores e regras socialmente compartilhados a respeito do ser adolescente (Senna \& Dessen, 2012). Na mesma direção, segundo a Organização Mundial da Saúde - OMS (1965), a adolescência é um fenômeno biopsicossocial que corresponde à segunda década da vida, isto é, dos 10 aos 20 anos ${ }^{1}$. Portanto, a adolescência deve compreendia como algo plural e não universal que transcende mudanças fisiológicas (puberdade) e que é influenciado por fatores culturais e históricos. Considerando o caráter relacional, contextual e dinâmico da adolescência, a concepção bioecológica é um sistema teórico-metodológico vantajoso para o estudo científico desse momento do ciclo vital.

0 estudo dos processos de resiliência na adolescência pode ser particularmente relevante quando ocorre simultaneamente com acontecimentos de vida que podem trazer prejuizos a diversos aspectos do desenvolvimento, como é o caso da exposição à violência sexual. De fato, segundo Masten (2014), a transição para a vida adulta é caracterizada por continuidades e descontuidades porque existem trajetórias de recuperação frente às adversidades que se iniciam durante a adolêscencia, podendo desencadear uma nova cadeia de oportunidades. Para a autora, essas mudanças nos padrões menos adaptativos são possíveis quando há a presença concomitante de recursos protetores internos e externos ao indíviduo, tais como competências cognitivas e sociais, presença de rede de apoio familiar e comunitário e políticas públicas de proteção ao desenvolvimento infantojuvenil.

Na Amazônia, apesar dos avanços da Rede de Proteção e do Sistema de Garantia de Direitos de Crianças e Adolescentes, a violência sexual contra crianças e adolescentes ainda é uma ferida aberta a sangrar, ferindo a dignidade humana e roubando a infância de tantas crianças e adolescentes. A violência sexual contra crianças permeia estruturas de poder, sejam políticas ou econômicas, em grande parte organizada e fortalecida de forma contínua, onde a impunidade ainda impera. Mas a violência sexual contra crianças e adolescentes na Amazônia brasileira é também naturalizada na cultura e uma nova mentalidade precisa ser instaurada. É ainda um fenômeno complexo, permeado de ambiguidades e contradições cuja compreensão requer a interpretação de suas múltiplas dimensões: a estrutural, a cultural, a legal (jurídica), a ética e a política (Fernandez, 2018).

A Política Nacional dos Direitos Humanos de Crianças e Adolescentes no Brasil, provocou, a partir de uma construção coletiva envolvendo o terceiro setor, a sociedade civil (com grande força dos movimentos sociais) e o Governo, a criação do Plano Nacional de Enfrentamento da Violência Sexual contra Crianças e Adolescentes (Brasil, 2015), existente desde 0 ano 2000, organizado em seis eixos estratégicos frente à violência sexual: 1) Prevenção; 2) Atenção; 3) Defesa e Responsabilização; 4) Participação e Protagonismo; 5) Comunicação e Mobilização Social; 6) Estudos e Pesquisas. A Cáritas, organismo da Igreja Católica, por meio do Projeto Içá Ação e Proteção, em consonância com Plano Nacional de Enfrentamento da Violência Sexual contra Crianças e Adolescentes, implantou em 2016 uma série de iniciativas voltadas para 0 enfrentamento da violência sexual contra crianças e adolescentes para os estados do Pará e Amazonas, ambos pertencentes a Amazônia brasileira.

\section{OBJETIVO DA INVESTIGAÇÃO}

0 presente estudo tem por objetivo apresentar a experiência do Projeto Içá-Ação e Proteção (Cáritas) na prevenção da violência sexual contra crianças e adolescentes na Amazônia brasileira, identificando fatores de resiliência em ações de protagonismo de adolescentes. Pretende-se, em particular, analisar os resultados relativos ao Eixo 4 - Participação e Protagonismo de crianças e adolescentes e ao Eixo 6 - Estudos e Pesquisas do referido projeto, que visamfortalecer a articulação, o conhecimento e a visibilidade das expressões do abuso, exploração sexual e tráfico de crian- 
ças e adolescentes na Amazônia brasileira por meio do protagonismo de crianças e adolescentes integrados à rede comunitária onde residem, à rede de proteção e ao sistema de garantia de direitos de crianças e adolescentes.

\section{PARTICIPANTES}

Trata-se de um relato de experiência das atividades desenvolvidas por 1.434 adolescentes participantes de um projeto de intervenção comunitária, Projeto Icá Ação e Proteção, em 19 cidades dos estados do Amazonas e Pará no período de 2016 a 2018. As cidades do estado do Pará onde se desenvolveram as ações foram: Belém Abaetetuba, Curralinho, Melgaço, Santarém, Óbidos, Alenquer, Oriximiná e Juruti. No Estado do Amazonas elegeram-se as cidades: Manaus, Careiro da Várzea, Careiro Castanho, Manaquiri, Iranduba, Manacapuru, Novo Airão, Presidente Figueiredo, Rio Preto da Eva, Itacoatiara.

\section{METODOLOGIA}

0 Eixo 4 - Protagonismo e Participação de Crianças e Adolescentes do Projeto (Brasil, 2013) propõe e exige: fomento de estratégias e mecanismos que facilitem a participação organizada e a expressão livre de crianças e adolescentes, em especial sobre os assuntos a eles relacionados, considerando sua condição peculiar de desenvolvimento, pessoas com deficiência e as diversidades de gênero, orientação sexual, cultural, étnico-racial, religiosa, geracional, territorial, nacionalidade e opção política. Tem como objetivo estratégico promover o protagonismo e a participação de crianças e adolescentes nos espaços de convivência e de construção da cidadania, inclusive nos processos de formulação, deliberação, monitoramento e avaliação das políticas públicas, ampliando 0 acesso de crianças e adolescentes, na sua diversidade, aos meios de comunicação para expressão e manifestação de suas opiniões.

Tendo presente as prorrogativas deste Eixo 4, a participação dos adolescentes no Projeto se deu por meio de proposta motivadora por contato pessoal em escolas, encontros de grupos de comunidades e igrejas. Esse despertar foi realizado pelos Agentes Cáritas, pertencentes ao ambiente ou próximos de onde vivem os adolescentes e suas famílias.

A partir do primeiro despertar em encontros específicos sobre violência sexual, foi apresentada aos adolescentes a possibilidade de constiturem partes integrantes do Projeto Içá Ação e Proteção. Em seguida, com a permissão de suas famílias e com a adesão voluntária dos adolescentes, seguiram-se as capacitações e as ações propriamente ditas com maior atenção para os mesmos atuarem no Eixo Prevenção, como resposta ao Eixo 4.

Inicialmente, neste eixo do Projeto Içá Ação e Proteção, foi estabelecido como objetivo ampliar a participação e protagonismo de 950 crianças e adolescentes na elaboração e execução de ações de proteção ao abuso, exploração sexual e tráfico de crianças e adolescentes para fins de exploração sexual. Esperava-se, como resultados, que pelo menos 38 ações de mobilização social fossem elaboradas e implementadas por crianças e adolescentes nas temáticas sobre 0 abuso, exploração e tráfico de crianças e adolescentes para o período de 2017 a 2019. Para esse estudo, elegeu-se um recorte para os adolescentes.

Por fim, como indicadores, para além do a) número de crianças e adolescentes envolvidos e do número de ações elaboradas e implementadas, era esperado que b) ao menos $70 \%$ de uma amostra aleatória das crianças e adolescentes participantes avaliassem a importância das ações de mobilização social como "muito alta" (1) ou "alta" (2) (em uma escala de 1 a 5) e que fossem realizadas 72 visitas de assessoria, monitoramento e apoio nas ações de mobilização; c) Ao menos 950 professores e lideranças comunitárias abordassem nas escolas e comunidades as temáticas do abuso, exploração e tráfico de crianças e adolescentes com base nas 38 capacitações. 


\section{VIOLÊNCIA SEXUAL CONTRA CRIANÇAS E ADOLESCENTES: RESILIÊNCIA E PROTAGONISMO NA AMAZÔNIA}

\section{RESULTADOS ALCANÇADOS}

0 Projeto Içá Ação e Proteção, pelo protagonismo dos adolescentes, desenvolveu as atividades que seguidamente se descrevem com respetivos resultados alcançados, que superam todas as metas traçadas nos objetivos iniciais.

Capacitações de Adolescentes, professores e lideranças comunitárias por meio de oficinas e rodas de conversa: atividades que visavam o fortalecimento do protagonismo de adolescentes em vista do conhecimento sobre: 0 acesso aos direitos garantidos por Lei, a autoproteção e participação na prevenção à violência sexual contra crianças e adolescentes, conhecimento sobre o Estatuto da Criança e do Adolescente (ECA), bullying, participação política; ética; liderança; tipos de violências, violência sexual; respeito; amizade-namoro; protagonismo juvenil; tráfico de pessoas; drogadição; comunidade - cultura-sociedade- família; comunicação e mídias; acesso seguro e autocuidado no uso das TICs; Respeito à diversidade (manifestações culturais amazônicas indígenas/ribeirinhos e quilombolas, afro-brasileiras, indígenas). Estas atividades recorreram sobretudo a estratégias didáticas de teatro, pintura, expressão corporal, dança, musicoterapia, confecção de vídeos por celular, fotografias por celular, gravuras e colagem em papel. Foram realizadas 74 capacitações (12 no Amazonas e 62 no Pará), com a participação de 1.434 adolescentes.

Ações de mobilização e interação com a rede de proteção e o sistema de garantia de direitos: Atividades de prevenção ao abuso, exploração sexual e tráfico de crianças e adolescentes para fins de exploração em Fórum de Saúde; Audiências Públicas; Fórum "Família Escola e Comunidade"; Semana do Estatuto da Criança e do Adolescente (Semaneca); Semana do 18 de maio; Grito dos Excluídos; Ações em Shopping Centers; Ações em comunidades ao longo dos rios, lagos, ilhas fluviais, igarapés e comunidades indígenas e de povos tradicionais; Ações em espaços religiosos. Foram realizadas, em Manaus,49 Ações de Mobilização, tendo como público alvo 2.008 pessoas, com controle por frequência/assinatura. No Pará foram realizadas 78 ações, com 12.125 participantes. Para além disso, foram realizadas ainda, em Manaus, 34 Rodas de Conversa com 4.126 pessoas (controle por frequência/assinatura) e no Pará 62, com 10.386 participantes.

Visitas domiciliares: foram realizadas visitas domiciliares às famílias dos adolescentes que participaram do Projeto que também visavam a convivência e o fortalecimento de vínculos que ajudam no desenvolvimento do protagonismo dos adolescentes. Encontros com os pais/responsáveis: foram realizadas mensalmente, com roda de conversa sobre temas voltados à realidade das famílias, ao fortalecimento de vínculos familiares e a temas que despertassem a autocritica, a participação e 0 protagonismo dos adolescentes. 0 Projeto Içá entende que no processo de participação e protagonismo dos adolescentes, a presença e o suporte de apoio às famílias dos adolescentes é de suma importância. No Amazonas foram realizadas 25 visitas, com um total de 680 pessoas atingidas. No Pará foram realizadas 98 visitas com um total de10.824 pessoas.

Visitas de Assessoria e monitoramento das ações (com planejamento e avaliações) nas escolas, com parceiros da Rede de Proteção, igrejas e comunidades: encontros de discussão e elaboração de atividades coletivas desenvolvidas pelos adolescentes e Agentes da Cáritas. Contou ainda com a participação de professores, gestores, alunos, líderes comunitários, ribeirinhos, líderes religiosos, pais/responsáveis e demais interessados na temática da violência sexual contra crianças e adolescentes. No Amazonas foram realizadas 38 Visitas e no Pará 65 visitas.

Avaliação dos próprios adolescentes sobre as atividades de capacitação/sensibilização nas rodas de conversa, junto aos professores, gestores e lideranças comunitárias.

Aplicação de instrumento próprio de avaliação escrito: foram aplicadas em uma amostra de 65 adolescentes. Em uma escala de 1-5 (muito alta, alta, média, baixa e baixíssima), utilizando 0 esquema básico de avaliação "Que bom, Que pena, Que tal”. Dos adolescentes que avaliaram $80 \%$ 
marcaram muito alta, $20 \%$ alta. Os pontos mais destacados foram: "Que bom" - as rodas de conversa ajudam a esclarecer os assuntos que ainda não conhecíamos e nos preparam para ajudar no enfrentamento da violência sexual; "Que pena" - nem todos foram assíduos aos encontros; "Que tal"- que em todas as rodas de conversa todos pudessem ter mais tempo para expressar suas opiniões e ideias. Esse foi um modelo de avaliação aplicado também para outras atividades, no entanto, para esse estudo não foram consideradas os outros percentuais.

Dos Relatórios oriundos das reuniões/encontros de avaliação das atividades junto aos parceiros da rede de proteção e comunidades constatou-se que:

1) Houve valorização e reconhecimento da cultura local e dos recursos do ambiente onde vivem os adolescentes. 2) Descobriu-se e se reconheceu adolescentes competentes nas diversas temáticas que the dizem respeito e com mais condições de se autoprotegerem e de protegerem outras crianças e adolescentes no que diz respeito às violações cotidianas sofridas. 3) Fortalecimento na participação de adolescentes no protagonismo das ações. 4) Credibilidade nas escolas para realizar 0 trabalho de prevenção e protagonismo. 5) Melhoria dos vínculos familiares para os adolescentes participantes do projeto. 6) Despertar e reconhecimento de talentos nas diversas expressões artísticos-culturais entre os adolescentes. 7) Participação de adolescente no planejamento das ações. 8) Aumento das denúncias de violência sexual nas localidades onde residem os adolescentes. 9) Participação de adolescentes em outras ações envolvendo violações de crianças e adolescentes sem a participação direta dos agentes Cáritas envolvidos no Projeto, mas por iniciativas locais próprias, envolvendo sobretudo, as escolas, comunidades e a Rede de Proteção local. 10) Melhoria da autoestima de adolescentes e alegria dos familiares quanto à participação protagonista no Projeto. 11) Fortalecimento e despertar de lideranças adolescentes em outras atividades nas escolas onde ocorreram as ações. 12) Publicação de material específico produzido por adolescentes na prevenção da violência sexual contra crianças e adolescentes. 13) Integração de ações envolvendo adolescentes e outras fases geracionais. 14) Desconstrução da ideia de "adolescente aborrecente" e "adolescente indolente", para adolescentes protagonista e sujeito de direitos. 15) Sentimento de pertença dos adolescentes frente à sua comunidade de origem e às escolas que frequentam. 16) Conhecimentos de contextos socioculturais e de alternativas de mudança frente às situações de riscos e de vulnerabilidade em que se encontram crianças e adolescentes a partir dos próprios adolescentes e de suas famílias. 17) Busca e (re)conhecimento dos recursos positivos existentes na própria comunidade onde residem os adolescentes. 18) Despertar de outras iniciativas para 0 desenvolvimento integral dos adolescentes nos grupos, escolas, igrejas e comunidades. 19) Propostas ao poder público partindo dos adolescentes quanto à garantia dos direitos de crianças e adolescentes assegurados pelo ECA e políticas públicas para a área: Fóruns, Conselhos, Audiências Públicas e outras instâncias de discussão sobre direitos da criança e do adolescente.

\section{DISCUSSÃO}

É importante superar a visão de resiliência como um agente abstracto e externo operador de prevenção às causas e consequências da violência sexual e ainda dos efeitos do empobrecimento e da miséria que muitas famílias vivem, inclusive na Amazônia. Tampouco se deve atribuir a violência sexual de crianças e adolescentes às consequências das condições de pobreza e miséria que elas e suas famílias se encontram, uma vez que esse tipo de violência ocorre em todas as culturas e sociedades e em todos os níveis socioeconômicos. No entanto, superando a ideia de determinismo e linearidade, crianças e adolescentes que vivem em condições socioeconômicas desvantajosas, isto é, em situação de pobreza e de miséria, estão mais expostas a todos os tipos de violações, sobretudo da exploração sexual. Uma vez que a garantia de seus direitos básicos não Ihes são garantidos, os seus corpos acabam muitas ou demasiadas vezes por serem negociados contra a própria 
vontade. Sujeitadas a um modo de sobreviver à dureza de vida que lhes foram impostas. Por outro lado, promover resiliência não é uma panaceia mágica substituta de formas políticas de combate à pobreza e miséria e, sobretudo, às desigualdades sociais (Junqueira \& Deslandes, 2003). Como sublinha Masten (2014), a resiliência é uma magia, mas uma magia relativamente comum que depende da presença e acionamento de recursos de proteção internos mas também externos à pessoa, ao longo do seu ciclo de vida. Momentos de transição ou pontos de viragem como a adolescência são particularmente propícios ao desenvolvimento de trajetórias de resiliência. Quando falamos de violência sexual contra crianças e adolescentes (abuso e exploração) a literatura especializada nos aponta, de um modo geral, que a quebra de confiança com a pessoa adulta pode ser resultante da violência sofrida. Poderia então parecer paradoxal pensar que uma criança ou adolescente fosse capaz de desenvolver resiliência a partir da participação de ações com pessoas adultas próximas, incluindo membros da família, uma vez que muitas vezes aqueles que deveriam protegê-las, são os que também cometem a violência sexual. Contudo, é importante construir relações de confiança que permitam compreender que nem todos os adultos abusam ou exploram sexualmente crianças e adolescentes. Para além disso, será também importante reconhecer que quem conhece mais sobre 0 que aconteceu e 0 que acontece no cotidiano das crianças e adolescentes, incluindo os casos de violação, são as próprias crianças e adolescentes. Crianças e adolescentes mais do que sujeitos passivos de uma reflexão ou de uma ação, são pessoas competentes para serem portavozes de si mesmas. São além de sujeitos de direitos, promotoras desses direitos. Será fundamental conseguir fomentar esse protagonismo sem recuar a responsabilidade dos adultos e do poder público em Ihes garantir desenvolvimento e proteção integral.

Em termos de prevenção no campo da saúde pública relacionado à díade resiliência e maus-tratos, prevenção e promoção, acredita-se que os fatores de resiliência podem estar presentes e promovidos antes, durante e após um abuso sexual sofrido quando a prevenção se torna tônica de um programa institucional ou comunitário proposto (Bouvoir,1999; Junqueira \& Deslandes, 2003; William, 2009). No entanto, programas e propostas de prevenção e intervenção não podem se fechar apenas no aspecto do autocuidado e da autoproteção, pois além de serem pouco eficazes na diminuição do risco, podem ainda reforçar a ideia de culpabilização, revitimando crianças e adolescentes que sofreram abuso ou exploração sexual.

A resiliência pode ser promovida por meio de relações de confiança, de incentivo, de apoio, com 0 reconhecimento de recursos próprios dos adolescentes, das pessoas e do ambiente onde esses se encontram, incluindo os valores culturais (Masten, 2014; Ungar, 2015; Wright \& Masten, 2015). Se assim for, o foco da atenção ao desenvolvimento e à saúde de crianças e adolescentes passa a ser assumido na perspectiva do cuidado (Junqueira \& Deslandes, 2003, Ribeiro, 2018). No mais, se resiliência pode ser compreendida ainda como processo dinâmico e interacional entre a pessoa e seu entorno, os acontecimentos ao longo da vida e os fatores de proteção, como afirma Munist et al.(1998), foi no Projeto Içá Ação e Proteção que a promoção de resiliência veio acompanhado de uma ação protagonista em conjunto na perspectiva de atitudes de cuidado comunitário e em rede.

0 Projeto Içá Ação e Proteção mostrou que ninguém se torna protagonista e sujeito da própria história sozinho, como também não existem sujeitos resilientes sozinhos e tampouco mudanças mais amplas (socioculturais, políticas, ambas com implicações para a área da infância e adolescência) alcançadas de forma isolada. Os adolescentes realizaram ações de protagonismo no envolvimento comunitário, que partiu de uma construção coletiva e participativa de um projeto comum já atuante em rede.

Quem então participou e ainda participa desse cuidado comunitário e em rede? Participaram e participam aquelas e aqueles que lutaram para que o Plano Nacional de Enfrentamento da Violência 
Sexual fosse implantado. Participaram os Agentes voluntários/as da Cáritas integrados à rede de proteção e ao sistema de garantia de direitos de crianças e adolescentes da Amazônia brasileira (Amazonas e Pará). Participaram as crianças e adolescentes com suas famílias, professoras, professores, pesquisadores de universidades locais, gestores de escolas, líderes comunitários e de associações, técnicos/as, promotores, delegados, parlamentares, líderes religiosos, lideranças indígenas e de comunidades ribeirinhas e tradicionais. 0 cuidado no Projeto Içá Ação e Proteção foi compreendido e assumido na perspectiva de participação e articulação democrática, com fins de promoção de cidadania.

Quando pessoas da comunidade e pessoas de diferentes campos do saber de atuação se juntam para uma ação coletiva, Montero (2004a) afirma que é possível desenvolver ações capazes de fomentar e manter o controle e poder que os indivíduos podem exercer sobre seu ambiente individual e social para solucionar problemas que os afetam e lograr mudanças nesses ambientes e na estrutura social, constituindo assim uma práxis psicossocial. Nessa linha de pensamento, Sawaia (1997) afirma que o objetivo da práxis psicossocial é firmar o exercício da autonomia e da criação no espaço coletivo, de modo a potencializar as ações individuais e coletivas em prol do bem comum e da felicidade particular. Assim, pesquisas acadêmicas, ações e programas que são capazes de envolver os comunitários em seu processo de construção e desenvolvimento, criam consciência pessoal e social ao mesmo tempo, uma vez que a pessoa elabora um saber sobre si e sobre a realidade e coletividade em que se insere, buscando mudanças para qualificar o meio em que vive e, consequentemente, a si mesma. A isso pode-se chamar de promoção de resiliência.

\section{CONCLUSÕES}

Reconhece-se que o Projeto Iça Ação e Proteção em consonância com o Plano Nacional de Enfrentamento da Violência Sexual contra crianças e adolescentes criou e fortaleceu espaços organizados de participação proativa de adolescentes, assegurando a oferta de formação política, visando uma incidência qualificada e efetiva desse público na formulação, monitoramento e avaliação das políticas, programas e ações voltadas para o enfrentamento do abuso e/ou exploração sexual e do tráfico de crianças e adolescentes para fins de exxploração sexual. Fometou ainda a garantia da construção da autonomia e da participação de adolescentes participação em de pesquisas e na implementação de estratégias de prevenção do abuso e/ou exploração sexual, com foco no acesso seguro e autocuidado no uso das TICs, incluindo ações de disseminação de informações sobre suas potencialidades e riscos, tanto em nível pessoal como sociocomunitário. 0 Projeto Iça Ação e Proteção criou ainda possibilidade de envolvimento dos adolescentes em atividades que envolveram a valorização das raízes e da cultura local e que promoveram a construção de valores de respeito à diversidade, promovendo o respeito à diversidade (manifestações culturais amazônicas - indígenas/ribeirinhos e quilombolas, afro-brasileiras).

As conclusões dessa experiência mostraram que resiliência não é sinônimo de invulnerabilidade e a sua promoção é imperativo necessário para um desenvolvimento mais saudável na infância e adolescência. Os resultados indicaram que crianças e adolescentes mais do que sujeitos passivos de qualquer ação ou programa, têm que ser assumidos/as como sujeitos de direito, de participação, decisão, de autonomia e de protagonismo participativo e transformador no enfrentamento do abuso e exploração sexual.

\section{REFERÊNCIAS BIBLIOGRÁFICAS}

Algeri, S., Souza, L. M. (2006). Violência contra crianças e adolescentes: Um desafio no cotidiano da equipe de enfermagem. Revista Latino Americana de Enfermagem, 14(4), 625-631. Retrieved from http://www.scielo.br/pdf/rlae/v14n4/pt_v14n4a23.pdf 


\section{VIOLÊNCIA SEXUAL CONTRA CRIANÇAS E ADOLESCENTES: RESILIÊNCIA E PROTAGONISMO NA AMAZÔNIA}

Antunes, C., \& Machado, C. (2013). A elaboração narrativa no abuso sexual: 0 papel das vítimas enquanto protagonistas no processo de mudança. Psicologia: Revista Da Associacao Portuguesa Psicologia, 27(1), 157-180

Bazon, M. R. (2009) Estudos epidemiológicos de maus-tratos com base nas informações colhidas junto ao setor educacional. In: L. C. Williams \& E. A Araújo (Orgs), Prevenção do Abuso Sexual Infantil: Um enfoque interdisciplinar. Curitiba: Juruá Editora.

Brasil. (2007a). Saúde de adolescentes e jovens. Caderneta. Retirado em 21/01/2011, de http://portal.saude.gov.br/saude/.

Brasil. (2007b). Lei no. 8069, de 13 de julho de 1990. Retirado em 20/12/2010, de http://www.planalto.gov.br/ccivil_03/Leis/L8069.htm.

Brasil (2013). Secretaria Especial de Direitos Humanos. Plano Nacional de Enfrentamento à Violência Sexual Contra Crianças e Adolescentes. Brasília, DF.

Bronfenbrenner, U., \& Morris, P. (1998). The ecology of developmental processes. EM W. Damon (Series Ed.) \& R. M. Lerner (Vol. Ed.), Handbook of child psychology. Vol. 1, Theoretical models of human development (pp. 993-1027). New York: John Wiley \& Sons.

Bouvoir, P. (1999). Abus sexuels et résilience. In: Souffrir et se Construire (M. - P. Poipot, org.), pp. 125-161, Ramonville: Editions Érès.

Cowan, P.A., Cowan, P. C., \& Schulz, M.S. (1996). Thinking about risk and resilience in families. In E. M. Hetherington \& E. A. Bleachman (Eds.), Stress, coping and resilience in children and families (pp. 1-38). New Jersey: Lawrence Erlbawm Associates.

Crawford, E., Wright, M. O. and Masten, A. (2006). Resilience and spirituality in youth. In E. C. Roehlkepartain, P. E. King, L. Wagener and P. L. Benson (Eds.), The handbook of spiritual development in childhood and adolescence (pp. 355-370). Thousand Oaks, CA: Sage

Deblinger, E., Runyon, M. K., \& Steer, R. A. (2014). Profiles of Personal Resiliency in Youth Who Have Experienced Physical or Sexual Abuse. Journal of Psychoeducational Assessment, 32(6), 558-566. Retrieved from http://search.ebscohost.com/login.aspx?direct=true\&AuthType=ip, uid \&db=eric \&AN=EJ1035539\&lang=pt-pt\&site=ehost-live \&scope=site

Fernandez, C.B. (2018). Enfrentamento da exploração sexual de crianças e adolescentes no Amazonas. In: Deslandes, S.F. \& Constantino, P.(Eds.) Exploração Sexual de crianças e adolescentes: interpretações plurais e modos de enfrentamento (315-349). São Paulo: UCITEC.

Gillespie, E. L., \& Panagioti, M. (2019). Childhood maltreatment and adult suicidality: a comprehensive systematic review with meta-analysis. Psychological Medicine, 49(7), 1057-1078

Habigzang, L; Caminha, R.M. (2004). Abuxo sexual contra crianças e adolescentes: conceituação e intervenção clínica. São Paulo: Casa do Psicólogo.

Habigzang, L.; Damásio, B.F.; Koller, S.H.(2013) Impact evaluation of a cognitive behavioral group therapy model for sexually abused girls. Journal of Child Sexual Abuse, v. 2, pp. 173-190.

Halpern, S. C., Schuch, F. B., Scherer, J. N., Sordi, A. O., Pachado, M., Dalbosco, C., ... Von Diemen, L. (2018). Child Maltreatment and Illicit Substance Abuse: A Systematic Review and Meta Analysis of Longitudinal Studies. Child Abuse Review, 27(5), 344-360.

Junqueira, M. F. P. \& Deslandes, S. F. (2003). Resiliência e maus tratos à criança. Cadernos de Saúde Pública, 19(1), 227-335.

Luo, T. E. (1998). Sexual abuse trauma among Chinese survivors. Child Abuse \& Neglect, 22(10), 1013-1026

Masten, A. S. (2014). Ordinary magic: Resilience in development. New York, NY, US: Guilford Press.

Miller, E. (2018). Reclaiming Gender and Power in Sexual Violence Prevention in Adolescence. Violence Against Women, 24(15), 1785-1793.

Moynihan, M., Pitcher, C., \& Saewyc, E. (2018) Interventions that Foster Healing Among Sexually 
Exploited Children and Adolescents: A Systematic Review, Journal of Child Sexual Abuse, 27:4, 403-423.

Monteiro, M. (2004). Introducción a lapsicologíacomunitaria: desarrollo,conceptos y processos. 1a ed. BuenosAires: Paidós.

Munist, M., Santos, H., Kotliarenco, M. A., Ojeda, E. N. S., Infante, F., \& Grotberg, E. (1998). Manual de identificación e promoción de la resiliencia [Versão eletrônica]. Washington, DC: Organización Panamericana de la salud.

Narvaz, M. G. \& Koller, S. H. (2004). 0 modelo bioecológico do desenvolvimento humano. In S. H. Koller (Ed.), Ecologia do desenvolvimento humano: Pesquisa e Intervenções no Brasil (pp. 5164). São Paulo: Casa do Psicólogo.

Organização Mundial da Saúde (1965). Problemas de la salud de la adolescencia. Informe de un comité de expertos de la O.M.S (Informe técnico n³08). Genebra.

Ribeiro, J. H. S (2013). Espaços Violados: uma leitura geográfica e psicossocial da violência sexual infanto-juvenil na área urbana de Manaus (2006-2010). Manaus: UEA Edições.

Ribeiro, J.H.S.; Costa, A. A.; Paião, L. S.; Morais, N. M. \& Costa, A. A. (2018)Violência sexual contra crianças e adolescentes: ideação suicida, tentativa de suicídio, automutilação e intervenção cognitivo-comportamental. In: Gutierrez, D. M. D. \& Ribeiro, J. H.S.(Eds.), Suicídio: diálogos interdisciplinares (pp. 313-340). Manaus: EDUA.

Sawaia, B. B. (1997). 0 ofício da psicologia social à luz da idéia reguladora desujeito: da eficácia da ação à estética da existência. In: Zanella, A.V. et al. (Eds.).Psicologia e práticas sociais (pp.3556). Porto Alegre: ABRAPSOSUL.

Senna, S. R. C. M., \& Dessen, M. A. (2012). Contribuições das teorias do desenvolvimento humano para a concepção contemporânea de adolescência. Psicologia: Teoria e Pesquisa, 28(1), 101108. Retrieved from http://www.scielo.br/pdf/ptp/v28n1/13.pdf

Silva, R. S.; Gonçalves, M. (2015) A ocorrência de transtornos psiquiátricos em crianças e adolescentes abusados sexualmente. UNICIÊNCIAS, v.19,n.1,p.72-78.

Theron, L. C., \& Phasha, N. (2015). Cultural pathways to resilience: Opportunities and obstacles as recalled by black South African students. In Youth resilience and culture: Commonalities and complexities, L. C. Theron, L. Liebenberg \& M. Ungar (eds), pp. 51 66. Dordrecht. Springer.

Ungar, M. (2015). Working with children and youth with complex needs: 20 skills to build resilience. New York, NY, US: Routledge/Taylor \& Francis Group.

Wright, M. 0., Crawford, E., \& Sebastian, K. (2007). Positive resolution of childhood sexual abuse experiences: The role of coping, benefit-finding and meaning-making. Journal of Family Violence, 22(7), 597-608

Williams, L. C.; Araújo, E. A.(2009) Prevenção do Abuso Sexual Infantil. Um enfoque interdisciplinar. Curitiba: Juruá Editora.

Wright, M. O. \& Masten, A. S. (2015) Pathways to resilience in context. In: Theron, L. C., Liebenberg, L., M. Ungar (eds) Resilience and culture. Commonalities and complexities (pp. 3-22) Dordrecht: Springer

10 Ministério da Saúde do Brasil (BRASIL, 2007a) adota a mesma faixa etária para delimitar a adolescência. Já para o Estatuto da Criança e do Adolescente - ECA (BRASIL, 2007b), a adolescência corresponde ao período entre 12 e 18 anos. 
